\title{
AVALIAÇÃO DO USO DA MORINGA OLEIFERA COMO BIOSSORVENTE NA REMOÇÃO DE CHUMBO DE ÁGUAS CONTAMINADAS
}

\author{
F. O. TAVARES ${ }^{1 *}$, L. A. D. M. PINTO ${ }^{2}$, T. R.T. SANTOS ${ }^{1}$, M. F. VIEIRA ${ }^{1}$, R. \\ BERGAMASCO $^{1}$, A. M. S. VIEIRA ${ }^{2}$ \\ ${ }^{1}$ Universidade Estadual de Maringá, Departamento de Engenharia Química \\ ${ }^{2}$ Universidade Estadual de Maringá, Departamento de Engenharia de Alimentos \\ e-mail: fernandaoliveiratavares@gmail.com
}

\begin{abstract}
RESUMO
A qualidade e a acessibilidade à água potável são de extrema importância, uma vez que ela é um dos elementos básicos para a sobrevivência humana e para o desenvolvimento das atividades econômicas. Porém a minimização dos recursos naturais e a elevada demanda tornam a escassez um fato iminente. A busca por novas alternativas e técnicas de recuperação de águas tem se tornando comum nos dias atuais. Dentre os compostos naturais estudados se destaca a Moringa oleifera. Deste modo, este trabalho teve por objetivo avaliar o percentual de remoção de chumbo da água utilizando sementes de Moringa oleífera como biossorvente, para isto foram avaliados: tempo de contato, influência da temperatura no processo, massa de adsorvente e $\mathrm{pH}$. Como resultado, o adsorvente mostrou grande potencial para a remoção de chumbo, podendo ser verificado através do elevado percentual de remoção obtido, de $96,55 \%$ à $25^{\circ} \mathrm{C}, \mathrm{pH} 5,5$ e agitação de $200 \mathrm{rpm}$, em um tempo de equilíbrio de 30 minutos. A análise da influência da temperatura e da quantidade de massa de moringa neste estudo mostrou que estes parâmetros não influenciaram significativamente no processo de remoção de chumbo, já o pH mostrou-se altamente significativo, apresentando percentual de remoção de apenas $17,62 \%$ em pH 1 e ao se elevar o pH para 6, a máxima remoção obtida alcança percentual superior a $98,8 \%$.
\end{abstract}

\section{INTRODUÇÃO}

O aumento das atividades industriais tem contribuído significativamente para o aumento da poluição ambiental e destruição dos ecossistemas (ARAÚJO et al. 2009), principalmente aquelas que apresentam resíduos que contenham metais pesados por serem prejudiciais devido aos seus efeitos tóxicos para os seres humanos e animais (TAGLIAFERRO et al. 2011).

Os metais pesados tornam-se tóxicos para os seres humanos quando as concentrações destes exceder os níveis permitidos. Com base na sua toxicidade a
Agência de Proteção Ambiental dos EUA (EPA) e a Organização Mundial da Saúde (OMS), estabeleceu o limite máximo admissível para íons de chumbo na água potável de 0,05 mg/L. Reconhecidos como contaminantes perigosos devido à sua elevada toxicidade, acumulação e retenção no corpo humano, nos seres humanos o chumbo pode provocar graves danos para os rins, sistema nervoso, sistema reprodutivo, fígado e para o cérebro (WANG et al. 2015).

A remoção de metais pesados da água envolvendo adsorção por materiais orgânicos e inorgânicos, tem se mostrado uma opção bastante interessante. A adsorção tem 
emergido como um processo muito eficaz na remoção dos metais. Para esta técnica, o material mais comumente utilizado é o carbono ativado, que é altamente eficiente em várias situações (DABROWSKI, 2000). No entanto, seu alto custo, muitas vezes faz com que este material seja inviável para uso prático. Por esta razão, vários adsorventes de baixo custo ganharam cada vez mais atenção (RAO, 2010).

A sorção de metais por resíduos agrícolas, material natural e subprodutos industriais pode ser atribuída à presença de grupos polares funcionais, como por exemplo de lignina, proteínas, hidratos de carbono, entre outros. Estes grupos têm a capacidade de se ligar aos metais pesados para formar complexos com os íons metálicos em solução (PAGNANELLI, 2003). As principais vantagens da biossorção sobre métodos de separação convencionais incluem o baixo custo, alta eficiência, minimização de produtos químicos e do lodo biológico, regeneração do biossorvente, possibilidade de recuperação de metal (REDDY, 2010).

A moringa é uma planta originária do nordeste da Índia. No Brasil, a princípio, a moringa foi introduzida para fins ornamentais e mais tarde veio a ser cultivada por sua importância medicinal e alto valor nutritivo, bem como por sua importância industrial devido as suas propriedades de coagulação, clarificação e adsorção em soluções aquosas na remoção de poluentes (MENEGHEL et al., 2013).

Dessa forma, o objetivo desse trabalho foi avaliar o percentual de remoção de chumbo da água utilizando sementes de Moringa oleífera como biossorvente, bem como determinação dos parâmetros de tempo de contato requerido para que o equilíbrio fosse atingido, além da influência da temperatura no processo de remoção.

\section{METODOLOGIA}

\subsection{Preparo do Adsorvente}

As sementes de moringa foram obtidas da cidade de Aracaju- SE, Brasil. Inicialmente, as sementes foram limpas e descascadas, em seguida, trituradas e desengorduradas seguindo a metodologia proposta por Martín et al. (2010) com modificações. Após trituradas, o pó obtido foi desengordurado com a adição de etanol a 95\% na proporção de 3:1, misturadas e levadas para agitação magnética por 45 minutos. Em seguida o sobrenadante foi separado por centrifugação a $3000 \mathrm{rpm}$ durante 45 minutos e o pellet formado foi seco em estufa à $60^{\circ} \mathrm{C}$ por 24 horas. Após a secagem, padronizou-se a granulometria a 32 mesh. O pó obtido do peneiramento foi utilizado como biossorvente.

A influência de todos os parâmetros no processo de remoção de chumbo por sementes de Moringa oleífera seguiu metodologia proposta por Seshaiah et al. (2011). Os estudos foram realizados em batelada e as soluções de chumbo utilizadas nos ensaios foram preparadas a partir de nitrato de chumbo. Todos os experimentos foram realizados em triplicata.

\subsection{Avaliação do Tempo de Equilíbrio e do Percentual de Remoção de $\mathbf{P b}^{2+}$}

Os estudos de biossorção foram realizados em batelada. Para determinação do tempo de equilíbrio $50 \mathrm{~mL}$ de chumbo a $4 \mathrm{mg} / \mathrm{L}$ foi adicionada à $0,5 \mathrm{~g}$ do adsorvente. $\mathrm{O} \mathrm{pH}$ utilizado foi o natural da solução, em torno de 5,5. O frasco selado foi colocado sob agitação a $200 \mathrm{rpm}$ e temperatura de $25^{\circ} \mathrm{C}$ em banho de agitação. $\mathrm{O}$ tempo de contato avaliado variou de 30 a 480 minutos. Em seguida, as suspensões foram retiradas da agitação e filtradas em membrana de $0,45 \mu \mathrm{m}$. A concentração de metais em solução foi analisada por meio de espectrometria por absorção atômica (AAS espectros, Varian AA, 55 Australia).

Para estimar o tempo de contato, o equilíbrio atingido foi reportado pela 


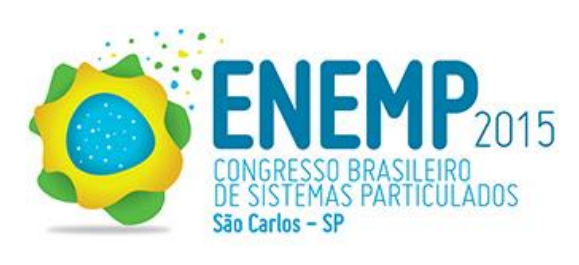

capacidade de adsorção do adsorvente, q (mg do metal/g do adsorvente), determinado com base na diferença de concentração do íon metálico, usando-se a Equação 01.

$\mathrm{q}=(\mathrm{Co}-\mathrm{Ce}) . \mathrm{V} / \mathrm{m}$

Nesta equação, Co é a concentração inicial de chumbo $(\mathrm{mg} / \mathrm{L})$; Ce a concentração final do chumbo no equilíbrio $(\mathrm{mg} / \mathrm{L}) ; \mathrm{V}$ o volume de solução (L) e $\mathrm{m}$ a massa do adsorvente $(\mathrm{g})$.

Já para a percentagem de remoção do íon metálico a partir da solução aquosa, utilizou-se a Equação 2.

Remoção \% $=\left(\left(\mathrm{C}_{\mathrm{o}}-\mathrm{C}_{\mathrm{f}}\right) / \mathrm{Co}\right) * 100$

Nesta equação, $C_{0}$ é a concentração inicial do metal $(\mathrm{mg} / \mathrm{L})$ e $\mathrm{C}_{\mathrm{f}}$ é a concentração residual de metal final após o período de adsorção.

\subsection{Efeito Da Temperatura}

Os ensaios foram realizados a partir de $50 \mathrm{~mL}$ de solução de chumbo a $4 \mathrm{mg} / \mathrm{L}$ adicionado de $0,5 \mathrm{~g}$ de adsorvente, a uma de rotação de $200 \mathrm{rpm}$ e pH 5,5. O tempo requerido para o ensaio foi proveniente do resultado obtido do tempo de contato para que o equilíbrio fosse atingido. As diferentes temperaturas avaliadas foram: 15, 25, 35 e 45 ${ }^{\circ} \mathrm{C}$. Para estimar o efeito da influência da temperatura, avaliou-se o percentual de remoção de íons metálicos a partir da solução aquosa, utilizando-se a equação 2 .

\subsection{Efeito Da Massa De Biossorvente}

$O$ efeito da massa de moringa no processo de biossorção foi realizado a partir de $50 \mathrm{~mL}$ de solução de chumbo a $4 \mathrm{mg} / \mathrm{L}$ adicionada de diferentes quantidades de massa $(0,5,0,7,1,0$ e $1,3 \mathrm{~g})$, com rotação de $200 \mathrm{rpm}$ e $\mathrm{pH} 5,5$. O tempo e a temperatura utilizada neste ensaio foram provenientes do resultado obtido dos experimentos de tempo de contato e temperatura. Para estimar o efeito da massa de adsorvente, o percentual de remoção de íons metálicos foi avaliado a partir da solução aquosa, utilizando-se a equação 2 .

\subsection{Efeito Do pH}

$\mathrm{O}$ estudo da influência do $\mathrm{pH}$ foi realizado a partir de $50 \mathrm{~mL}$ de solução de chumbo a $4 \mathrm{mg} / \mathrm{L}$ em diferentes $\mathrm{pHs}$ $(1,3,5,6,7,8,10$ e 12), submetidas a uma agitação de $200 \mathrm{rpm}$. O tempo, a temperatura e a massa de adsorvente utilizada no ensaio foram selecionadas a partir dos resultados obtidos neste estudo. Para estimar o efeito do $\mathrm{pH}$ na remoção de $\mathrm{Pb}^{2+}$, o percentual de remoção foi avaliado utilizando-se a Equação 2.

\section{RESULTADOS E DISCUSSÃO}

\subsection{Avaliação do Tempo de Equilíbrio e do Percentual de Remoção de $\mathbf{P b}^{\mathbf{2 +}}$}

A Figura 1 correlaciona os tempos avaliados no ensaio com a concentração residual de chumbo obtida, bem como com o percentual de remoção de chumbo e com a capacidade de remoção da moringa.

Figura 1- Capacidade absortiva das sementes de moringa em função do tempo.

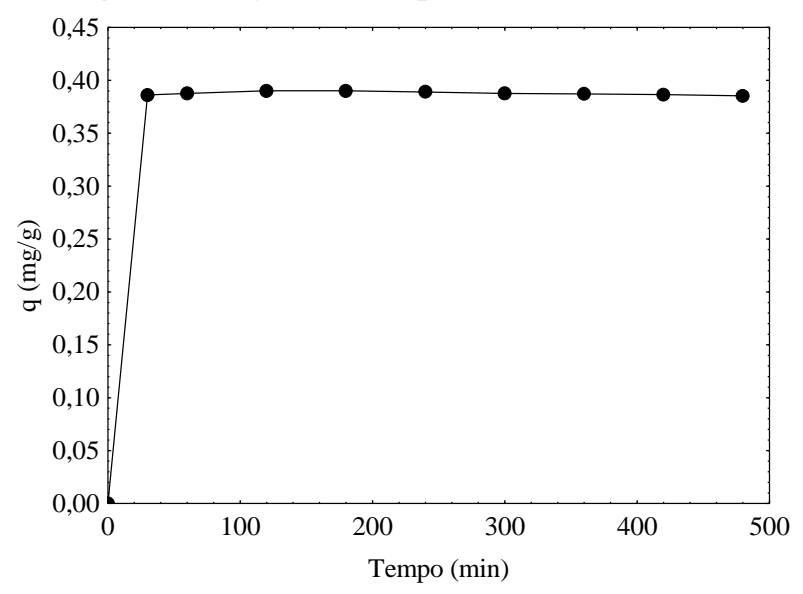

A partir da figura 1, pôde-se observar a influência do tempo na remoção de chumbo, sendo possível avaliar o tempo em que ocorre 


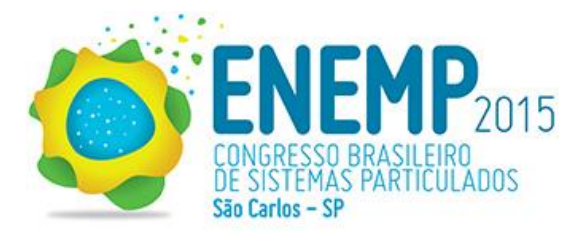

o equilíbrio. A análise dos tempos avaliados não apresentou diferença significativa ao nível de $5 \%$.

Analisando a figura 1, para a concentração de metal estudada de $4 \mathrm{mg} / \mathrm{L}$, é possível verificar que a biossorção aumentou rapidamente durante os primeiros $30 \mathrm{~min}$ e após manteve-se praticamente constante. Este comportamento pode ser atribuído a saturação dos sítios disponíveis presentes na moringa para ocorrência da adsorção.

O percentual de remoção de chumbo no ensaio realizado variou de 96,35 a 97,56 para os tempos variando entre 30 a $480 \mathrm{~min}$, porém os resultados não apresentaram diferença significativa ao nível de 5\%, sendo o percentual de remoção atingido no equilíbrio de $96,55 \%$.

O tempo de contato necessário para que o sistema atinja o equilíbrio depende da natureza do biossorvente e quantidade de locais de adsorção disponíveis. (MENEGHEL, 2013). Assim como determinado neste estudo, Reddy et al. (2010) em seu trabalho usando casca de Moringa oleífera como biossorvente para a remoção de chumbo, determinou como tempo de contato para se atingir o equilíbrio na remoção de chumbo 30min, já para o uso da folha como biossorvente, sob as mesmas condições $\left(\mathrm{pH} 5,25^{\circ} \mathrm{C}, 10 \mathrm{mg} / \mathrm{L} \mathrm{Pb}^{2+}\right.$ e $0,4 \mathrm{~g}$ de adsorvente) o tempo de equilíbrio foi superior, em torno de 50min.A determinação do tempo de equilíbrio usando sementes de moringa, também foi avaliada para outros metais, dentre eles, o Cádmio e o Cromo, em estudos desenvolvidos por Sharma et al. (2006) e Meneghel et al. (2013), respectivamente, em que os tempos de equilíbrio foram superiores ao encontrado neste estudo. Para o cádmio, o tempo suficiente para que ocorresse 0 equilíbrio foi de 40 minutos para uma solução a $10 \mathrm{mg} / \mathrm{L}$ adicionado de $2 \mathrm{~g}$ de biossorvente, enquanto que para o cromo foi de 120 minutos utilizando-se uma solução com a mesma concentração, porém diferindo na quantidade de moringa, $400 \mathrm{mg}$.

\subsection{Efeito Da Temperatura}

A Figura 2 apresenta a influência da temperatura no processo de adsorção de chumbo sobre o biossorvente moringa.

Figura 2- Decréscimo da remoção de chumbo em função da temperatura de biossorção.

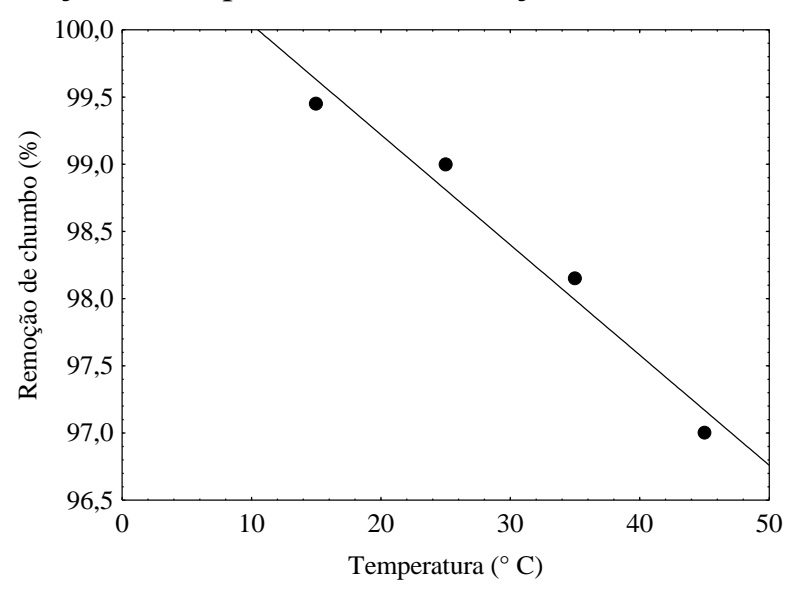

A temperatura consiste em um dos parâmetros que podem influenciar de maneira significativa no processo de adsorção. De acordo com a figura 2, é possível verificar que o aumento da temperatura ocasionou um decréscimo da remoção de chumbo, variando de 99,45 para $97 \%$ quando a temperatura variou de 15 para $45^{\circ} \mathrm{C}$. A pequena diferença obtida faz com que seja vantajoso, por ser economicamente mais viável, o uso da temperatura mais próxima à ambiente para o processo, mantendo-se a remoção elevada sem que seja preciso alterar a temperatura do processo. Kumar et al. (2012) em seu estudo da biossorção de $\mathrm{Cd}$ (II) com casca de castanha de caju, verificou que a remoção decresceu de 80,13 para $74,32 \%$ quando a temperatura variou de 30 para $60^{\circ} \mathrm{C}$. O mesmo pode ser observado no estudo desenvolvido por ElSayed et al. (2011) em seu trabalho com o $\mathrm{Zn}(\mathrm{II}), \mathrm{Cd}(\mathrm{II})$ e $\mathrm{Mn}$ (II) usando o caule do milho, onde pôde observar uma diminuição da remoção variando de 52 para $28 \%$ para o $\mathrm{Zn}$, de 34 para $16 \%$ para o Cd e de 39 para $13 \%$ 


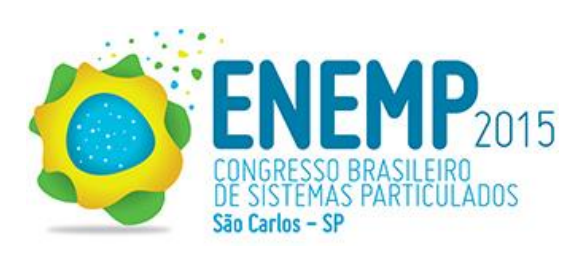

para o Mn quando a temperatura sofreu um aumento de $25^{\circ} \mathrm{C}$ para 55. Já em estudos realizados por Park et al. (2010), foi sugerido que o aumento da temperatura resulta numa melhora da remoção de metais devido a um aumento na sua atividade de superfície e energia cinética. Tal fato também pôde ser observado nos estudos realizados por GarcíaRosales e Colín-Cruz (2010) e Banerjee et al. (2012), em que os primeiros avaliaram a remoção de $\mathrm{Pb}$ (II) e $\mathrm{Cd}(\mathrm{II})$ usando talo de milho e observaram que o aumento da temperatura de 20 para $40^{\circ} \mathrm{C}$, resultou em 1,8 vezes a remoção dos compostos avaliados. Já Banerjee em seu estudo pôde avaliar o aumento na remoção de $\mathrm{Cu}$ (II) em função do aumento da temperatura fazendo-se o uso de casca de melância.

\subsection{Efeito Da Massa De Biossorvente}

A Figura 3 correlaciona as diferentes massas de moringa utilizadas com o percentual de remoção de chumbo.

Figura 3- Aumento da remoção de chumbo em função da massa de moringa.

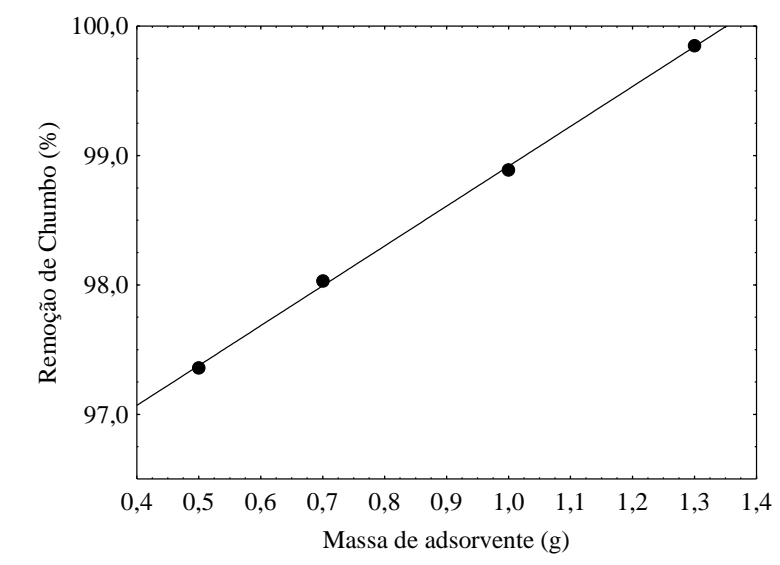

A partir da figura 3, é possível concluir que o aumento da quantidade de massa utilizada propicia um leve aumento da remoção. Neste estudo, o percentual de remoção variou de 97,36 a $99,85 \%$ quando variou-se a massa de 0,5 a $1,3 \mathrm{~g}$. Como o ganho obtido no processo de adsorção foi pequeno, o uso da menor massa de moringa seria justificado para se obter elevado percentual de remoção. Assim como neste trabalho, Sumathi, T. \& Alagumuthu, G. (2014) ao avaliar a influência da dose de adsorvente em seu estudo visando a remoção do arsênio a partir de Moringa Oleífera ativada, observou que o aumento da dose de adsorvente de $0,2 \mathrm{~g}$ até $1,2 \mathrm{~g}$ atingiu o máximo de remoção com o uso de 1,2 g, devido a existência de uma grande área de superfície gerando o maior número de ligações com o metal. Reddy et al. (2011) usando cascas de moringa como biossorvente na remoção de níquel, assim como neste estudo, observou o aumento do percentual de remoção quando aumentava-se a dose de adsorvente. Em seu estudo a variação da dose mostrou-se crescente para o percentual de remoção com a variação de 0,1 a $0,4 \mathrm{~g}$, porém ao aumentar a quantidade de massa do adsorvente até $0,8 \mathrm{~g}$ o percentual de remoção tornou-se praticamente constante. Resultado similar é obtido por Sharma et al.(2006) ao variar a dose de $2 \mathrm{~g}$ a $6 \mathrm{~g}$, onde existe um aumento gradual da remoção até atingir $4 \mathrm{~g}$ e depois, a remoção permanece constante.

\subsection{Efeito Do pH}

A Figura 4 correlaciona o percentual de remoção de chumbo com o $\mathrm{pH}$.

Figura 4 - Remoção de chumbo em função do pH.

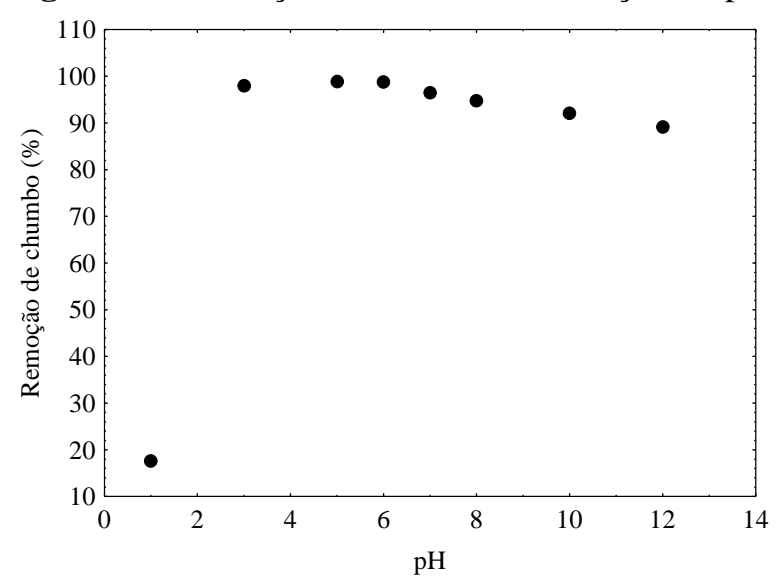

Analisando a figura 4, conclui-se que o uso de $\mathrm{pH} 1$ afetou significativamente a 


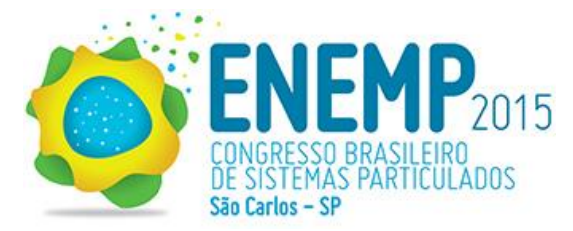

remoção de chumbo, havendo uma baixa remoção de chumbo, de apenas de $17,62 \%$. O aumento do $\mathrm{pH}$ de 3 a 6 conforme apresentado na figura, apresenta a remoção máxima obtida neste estudo, sendo esta superior a 98\%. Embora a diferença no percentual obtido não seja significativa, a escolha pelo $\mathrm{pH}$ 5-6 justifica-se pelo $\mathrm{pH}$ natural da solução $(\mathrm{pH} 5,5)$ não havendo necessidade de ajuste para promoção da máxima remoção. $\mathrm{O}$ aumento do pH a partir de 7 até 12, associa-se a redução da remoção de chumbo, onde o percentual de remoção obtido decresce de 96,45 para $89,14 \%$.

Taha et al. (2011) investigaram a influência do $\mathrm{pH}$ na adsorção do $\mathrm{PB}(\mathrm{II}), \mathrm{CD}$ (II) e Zn(IV) usando cascas de batata e assim como neste estudo, concluíram que a eficiência da remoção aumentava quando o $\mathrm{pH}$ aumentava de 2 a 6 e em pH acima de 6 a eficiência decrescia. Resultados similares foram observados por Reddy et al.(2011) na remoção de níquel usando casca de moringa oleífera. Feng et al. (2011) ao avaliar o percentual de remoção do $\mathrm{Pb}(\mathrm{II}), \mathrm{Cd}(\mathrm{II})$ e $\mathrm{Ni}(\mathrm{II})$ por cascas de laranja modificada concluíram que o aumento do $\mathrm{pH}$ de 2 para 5,5 aumentava o percentual de remoção. Assim como mostrado nesse estudo, ao variar o $\mathrm{pH}$ de 1 para 6, Kong et al (2014) avaliando a remoção de $\mathrm{Cu} 2+$, $\mathrm{Cd} 2+$ e $\mathrm{Pb} 2+$ usando bagaço de cana como adsorvente concluiu que a remoção é altamente dependente do $\mathrm{pH}$, onde o aumento do $\mathrm{pH}$ de 1 para 6 promove o aumento da capacidade de remoção de 19 para $213 \mathrm{mg} / \mathrm{g}$ para o cobre, 1 para $232 \mathrm{mg} / \mathrm{g}$ para o Cd e de 36 para $246 \mathrm{mg} / \mathrm{g}$ para o chumbo.

\section{CONCLUSÃO}

Como conclusão deste trabalho, pode-se dizer que as sementes de Moringa Oleífera utilizadas como biossorvente, exibem alto potencial de remoção de chumbo da água, apresentando remoção superior a $96 \%$ ao atingir o equilíbrio. De acordo com a variação XXXVII ENEMP
18 a 21 de Outubro de 2015 Universidade Federal de São Carlos da temperatura entre 15 e $45^{\circ} \mathrm{C}$ e da dose de adsorvente entre 0,5 a $1,3 \mathrm{~g}$, pôde-se concluir que estes parâmetros não influíram de maneira significativa ao nível de $5 \%$ para a remoção de chumbo à concentração de $4 \mathrm{mg} / \mathrm{L}$, porém o uso da temperatura próxima a ambiente e de uma menor quantidade de massa de adsorvente seria justificável para se obter uma máxima remoção aliado a viabilidade de aplicação do processo em grande escala. As análises referentes ao $\mathrm{pH}$, mostraram forte dependência da adsorção com este parâmetro, apresentando remoção de $17,62 \%$ para o pH 1 e elevado aumento da remoção ao elevar o pH para 6 . Mais estudos ainda são necessários para se elucidar completamente o mecanismo de adsorção de chumbo pela Moringa Oleífera, porém diante dos resultados apresentados, a moringa mostra-se com grande potencial na remoção de chumbo.

\section{REFERÊNCIAS}

ARAÚJO, C.S.T. Desenvolvimento de metodologia de analítica para extração e pré-concentração de Ag (I) utilizando a Moringa Oleífera Lam. 2009. 215 p. Tese de doutorado. Universidade Federal de Uberlândia - MG, 2009.

BANERJEE, K., RAMESH, S.T., GANDHIMATHI, R., NIDHEESH, P. V. and BHARATHI, K.S. A novel agricultural waste adsorbent, watermelon shell for the removal of copper from aqueous solutions. Iranica $\mathbf{J}$. Energy Environ, v. 3, p. 143-156, 2012.

DABROWSKI, A.; Adv. Colloid Interface Sci. 2000, 93, 135.

EL-SAYED, G. O., DESSOUKI, H. A. AND IBRAHIEM, S. S. Removal Of Zn(II), Cd(II) And $\mathrm{Mn}(\mathrm{II})$ From Aqueous Solutions By Adsorption On Maize Stalks. The Malaysian Journal of Analytical Sciences, 15, 21, 2011. 


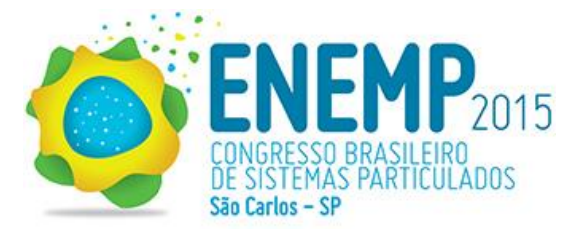

Environmental Protection Agency, National Primary Drinking Water Regulation, Washington, 2002.

FENG, N., GUO, X., LIANG, S., ZHU, Y., LIU, J. Biosorption of heavy metals from aqueus solutions by chemically modified Orange peel. J. Hazerd Mater, v.185, p.4954, 2011.

GARCÍA-ROSALES, G., COLÍN-CRUZ, A. Biosorption of lead by maize (Zea mays) stalk sponge. J. Environ. Manage, v. 91, p. 20792086, 2010.

KONG, W., REN, J., WANG, S., CHEN, Q. Removal of Heavy Metals from Aqueous Solutions Using Acrylic - modified Sugarcane Bagasse-based Adsorbentes: Equilibrium and Kinetic Stauies. BioResources, v.9, n. 2, p. 3184-3196, 2014.

KUMAR, P. S., RAMALINGAM, S., SATHYASELVABALA, V., KIRUPHA, S. D., MURUGESAN, A., SIVANESAN, S. Removal of $\mathrm{Cd}$ (II) from aqueous solution by agricultural waste cashew nut shell. Korean J. Chem. Eng., v. 29, p. 756-768, 2012.

MARTÍN, J. S.; GHEBREMICHAEL, K.; HEREDIA, J. B. Comparison of single-step and two-step purified coagulants from Moringa oleifera seed for turbidity and DOC removal, Bioresource Technology, v. 101, p. 6259- 6261, 2010.

MENEGHEL, A. P., GONÇALVES, A. C., STREY, L., RUBIO, F., SCHWANTES, D., CASARIN, J. Biosorption and removal of chromium from water by using moringa seed cake (Moringaoleífera Lam.). Química Nova, v. 36, p. 1104-1110, 2013.

MENHEGEL, A.P.; GONÇALVES, Jr. A.C.; RUBIO, F.; DRAGUNSKI, D.C.; LINDINO, C.A.; STREY, L. Biosorption of cadmium from water using moringa (Moringa oleifera Lam.) seeds. Water, Air e Soil Pollution. v. 224, p. 1383-1396, 2013.

PAGANANELLI, F., MAINELLI, S., VEGLIO, F., TORO, L. Heavy metal removal by olive pomace: biosorbent characterization and equilibrium modeling. Chem. Eng. Sci, v. 58, p. 4709-4717, 2003.

PARK, D., YUN, Y. S., PARK, J. M. The past, present, and future trends of biosorption. Biotechnol. Bioprocess. Eng., v. 15, p. 86$102,2010$.

RAO, R. A. K.; REHMAN, F.; J. Hazard Mater. 2010, 181, 405

REDDY, D. H. K. \& SESHAIAH, K. et al. Biosorption of $\mathrm{Pb}^{2+}$ from aqueous solutions by moringa oleífera bark: Equilibrium and Kinetic studies. Journal of Hazardous Materials, v. 174, p. 831-838, 2010.

REDDY, D. H. K., HARINATH, Y., SESHAIAH, K., REDDY, A. V. R. Biosorption of $\mathrm{Pb}$ (II) from aqueous solutions using chemicaly modified Moringa oleífera tree leaves. Chemical Engineering Journal, v. 162, p. 626-634, 2010.

REDDY, D. H. K., HARINATH, Y., SESHAIAH, K. REDDY, A. V. R., Biosorption of Ni(II) from aqueous phase by MoringaOleifera bark, a low cost biosorbent. Desalin., v.268, p. 150-157, 2011.

SHARMA, P., KUMARI, P., SRIVASTAVA, M. M., SRISVATAVA, S. Removal of cádmium from aqueous solutions sistem by shelled Moringa oleífera Lam. Seed powder. Bioresource Technology, v.97, p. 299-305, 2006.

SUMATHI, T. \& ALAGUMUTHU, G. Adsorption studies for arsenic removal using 
activated Moringa Oleifera. International Journal of Chemical Engeneering, v. 2014, 2014.

TAGLIAFERRO, G. V., PEREIRA, P. H. F., RODRIGUES, L. A., SILVA, M. L. C. P. et al. Adsorção de chumbo, cádmio e prata em óxido de nióbio (v) hidratado preparado pelo método da precipitação em solução homogênea. Quim. Nova, Vol. 34, No. 1, 101-105, 2011

TAHA, G. M., ARIFIEN, A.E., EL-NAHAS, S. Removal efficiency of potato peels as a new biosorbent material for uptake of $\mathrm{Pb}$ (II), Cd(II) and $\mathrm{Zn}$ (II) from the aqueous solutions. J. Solid Waste Technol. Manage, v.37, p. 128-140, 2011.

WANG, J., XIA, S., YU, L. Structure and bonding nature of $[\mathrm{PbCl}]^{+}$adsorption on the kaolinite (001) surface in aqueous system, Applied Surface Science, v.330, p.411417,2015.

World Health Organization, WHO Guidelines for Drinking Water Quality, Geneva, Switzerland, v. 1 and 2, 1981.

\section{AGRADECIMENTOS}

O desenvolvimento do trabalho contou com o apoio da Universidade Estadual de Maringá e com o suporte financeiro oferecido pela CAPES e Fundação Araucária. Sinceros agradecimentos às instituições citadas pelo incentivo a pesquisa. 